\title{
Miranda
}

Revue pluridisciplinaire du monde anglophone /

Multidisciplinary peer-reviewed journal on the English-

speaking world

13 | 2016

Thomas Spence and his Legacy: Bicentennial

Perspectives

\section{La notion de « responsabilité » dans le discours politique américain de 1980 à 2010 : le transfert rhétorique et idéologique des Républicains aux Démocrates}

\section{Lea Stephan}

\section{(2) OpenEdition \\ Journals}

Édition électronique

URL : http://journals.openedition.org/miranda/9710

DOI : 10.4000/miranda. 9710

ISSN : 2108-6559

Éditeur

Université Toulouse - Jean Jaurès

Référence électronique

Lea Stephan, «La notion de « responsabilité » dans le discours politique américain de 1980 à 2010 : le transfert rhétorique et idéologique des Républicains aux Démocrates », Miranda [En ligne], 13 | 2016 mis en ligne le 23 novembre 2016, consulté le 16 février 2021. URL : http://journals.openedition.org/ miranda/9710; DOI : https://doi.org/10.4000/miranda.9710

Ce document a été généré automatiquement le 16 février 2021.

Miranda is licensed under a Creative Commons Attribution-NonCommercial-NoDerivatives 4.0 International License. 


\title{
La notion de « responsabilité » dans le discours politique américain de 1980 à 2010 : le transfert rhétorique et idéologique des Républicains aux Démocrates
}

\author{
Lea Stephan
}

La mise en place de la Great Society, le programme de politiques intérieures et de politiques sociales du président Lyndon B. Johnson en 1965, s'est appuyée sur une forte idée de redistribution de la richesse. Le déclin de l'économie durant les années 1970, en revanche, a significativement fait diminuer la volonté des contribuables à voir utiliser leurs impôts pour financer des programmes sociaux. ${ }^{1}$ Cette baisse d'enthousiasme pour les programmes sociaux destinés aux pauvres est due, d'un côté, au sentiment des classes moyennes d'être trop fortement sollicitées fiscalement, ${ }^{2}$ et, de l'autre, au manque apparent de résultats. En effet, durant les années 1970, les dépenses pour les programmes sociaux ont augmenté. En revanche, le taux de criminalité, le taux de chômage, la toxicomanie, le nombre d'enfants nés hors mariage, ainsi que le recours aux aides sociales et autres allocations ont aussi augmenté. ${ }^{3}$ Cette émergence d'un quart-monde ou sous-prolétariat (underclass), majoritairement noir, a même fait oublier les progrès réels qui ont été faits. Ces progrès sont visibles dans l'expansion de la classe moyenne noire. ${ }^{4}$ Mais de façon beaucoup plus importante, cette underclass a favorisé l'émergence de l'idée propagée par les Républicains d'un gaspillage d'argent public pour des mesures inefficaces, ainsi que la vision d'un monde où certains travaillent dur pour que d'autres restent chez eux à ne rien faire, et ce dans un climat de perte de valeurs morales. ${ }^{5}$ Cette perception a amené une partie de la population à ne plus vouloir payer d'impôts pour des programmes sociaux. ${ }^{6}$ De plus, ce discours conservateur véhiculé par les Républicains joue implicitement sur des tensions raciales, car les contribuables sont identifiés comme la classe moyenne et ouvrière blanche, alors que les bénéficiaires de politiques sociales sont désignés comme faisant partie de 
la underclass noire. Ces derniers sont largement perçus comme des pauvres qui ne méritent pas l'aide sociale, car ils sont vus comme ne faisant pas assez d'efforts pour se sortir de la misère. ${ }^{7}$ Cette division raciale concernant les politiques sociales a empêché une alliance de classes sociales basée sur des intérêts communs. ${ }^{8}$

2 Ce climat économique et social a permis au Parti républicain d'instaurer un discours et une politique durable prônant la réduction des dépenses sociales et donc une diminution de l'intervention de l'Etat en faveur de plus de responsabilité personnelle. La notion de responsabilité personnelle est fermement ancrée dans la culture et la politique américaine. Des écrivains tels que Ralph Waldo Emerson ont largement contribué à diffuser une vision hautement individualiste de la réussite. ${ }^{9} \mathrm{La}$ responsabilité personnelle tient aussi une place cruciale dans le concept du Rêve Américain, accepté autant par la communauté noire que blanche. ${ }^{10} \mathrm{En}$ effet, alors que l'utilisation politique de la notion de responsabilité personnelle est habituellement associée aux conservateurs blancs, cette notion est depuis longtemps utilisée dans la communauté noire, par des penseurs conservateurs et libéraux (dans le sens américain du terme), intégrationnistes et séparatistes.

3 La notion de responsabilité est utilisée par des personnes d'horizons très différents, mais dans sa forme la plus stricte elle se réduit à une conception simple: c'est à l'individu seul, avec éventuellement l'aide de sa famille, de subvenir à ses besoins, sans compter sur l'aide de l'Etat. Il est donc facile d'utiliser la notion de responsabilité personnelle dans un discours qui justifie le désengagement de l'Etat en matière de politique sociale, stratégie principale des conservateurs et du Parti républicain depuis les années 1980 et l'élection de Ronald Reagan. ${ }^{11}$ Comment les Démocrates ont-ils fait face depuis lors à ce discours de responsabilité personnelle dans leur volonté d'expansion des politiques sociales?

4 Afin de répondre à cette question, il sera montré d'abord comment le discours conservateur de la responsabilité personnelle a été développé et propagé par le Parti Républicain, puis comment les idéologies et le discours se sont hybridées durant les mandats de Bill Clinton et George W. Bush, pour finalement voir comment Barack Obama parvient à utiliser la notion de responsabilité à son avantage.

\section{Les partis politiques et leur attitude vis-à-vis des politiques sociales}

5 En ce qui concerne les politiques sociales, des associations particulièrement étroites sont traditionnellement établies entre une certaine idéologie et le parti politique qui la défend.$^{12}$ Bien que le Parti démocrate ait commencé à adopter un discours ciblé sur la pauvreté dans les années 1930 et qu'il ait intégré la défense des droits civiques et droits pour les minorités dans le programme de 1948, l'association forte du parti avec les minorités raciales s'est vraiment faite durant les années $1960 .{ }^{13}$ Cette association s'est renforcée lors de l'élection présidentielle de 1964, qui marque le moment où les partis se sont positionnés clairement concernant la question de l'intervention de l'Etat fédéral pour aider les minorités raciales, avec le Parti démocrate en faveur d'une telle intervention, et le Parti républicain contre celle-ci. ${ }^{14}$ Selon Laura Katz Olson le Parti démocrate est toujours fortement associé aux politiques sociales, et ce malgré les années Clinton. ${ }^{15}$ Une plus forte polarisation a aussi été constatée au niveau législatif avec une division assez nette en fonction des partis. ${ }^{16}$ Une identification progressive 
s'est opérée, le Parti républicain étant associé avec une idéologie conservatrice et le Parti démocrate avec une idéologie libérale. ${ }^{17}$ Cette polarisation partisane et idéologique se révèle particulièrement autour de la question des impôts et des politiques sociales. ${ }^{18}$ Avant les années Clinton, on avait assisté à une opposition assez nette entre libéraux et conservateurs, entre les Démocrates fidèles à la tradition de la Great Society et les Républicains fervents défenseurs du "self-help». Cornel West a d'ailleurs noté la "paralysie politique » qui en découlait et il a déploré la rigidité intellectuelle des deux positions. ${ }^{19}$ Toutefois, ces observations avaient été faites avant les mandats de Clinton et sa tentative d'une nouvelle approche de la question. On peut donc constater jusqu'à la présidence de Clinton un certain recoupement entre partis et idéologies concernant la question des politiques sociales, ainsi que les problèmes des impôts, de la responsabilité, et du rôle de l'Etat qui y sont associés. Le problème avec ce genre d'associations est qu'elles se montrent parfois très durables. Le sénateur John McCain avait notamment essayé lors de la campagne électorale de 2008 de discréditer Obama en le baptisant « redistributeur en chef $» .{ }^{20}$ Cela montre deux choses : la facilité avec laquelle ce discours est ravivé et la continuité de l'image péjorative de la redistribution vers le bas et donc des politiques sociales.

6 Il y a une dimension raciale indéniable à cette question, bien que certains chercheurs considèrent que, dans le débat contemporain, la question du rôle de l'Etat prime sur la dimension raciale du discours. ${ }^{21} \mathrm{Je}$ ne partage pas cette interprétation. En revanche, j'adhère à la thèse selon laquelle le discours sur le rôle de l'Etat sert de code pour camoufler un discours raciste. ${ }^{22}$

Depuis les années 1970s, le facteur racial a été utilisé de deux manières par les conservateurs et le Parti républicain. Le premier but était une récupération d'une partie de l'électorat traditionnel démocrate, la classe ouvrière et moyenne blanche, les fameux Reagan-Democrats. Les Républicains ont joué sur la perception selon laquelle le Parti démocrate se préoccupe plus des minorités raciales que des Blancs issus de ces classes sociales. Les sous-entendus raciaux sont particulièrement efficaces sur les électeurs démocrates, qui soutiennent par principe idéologique plus d'intervention gouvernementale, mais peuvent en être dissuadés par un discours racial. ${ }^{23}$ De plus, ayant un programme économique peu compatible avec les intérêts de la classe ouvrière et moyenne blanche, le Parti républicain a investi le plan des valeurs culturelles, en soutenant des positions tranchées sur l'avortement, l'éthique du travail, ou encore la responsabilité personnelle. ${ }^{24}$

\section{La responsabilité selon les Républicains}

L'élection de Ronald Reagan en 1980 marque un tournant important. Le programme du Parti républicain est devenu bien plus conservateur pour cette élection ${ }^{25}$ qui marque aussi le début de la discréditation ouverte des revendications raciales et du libéralisme. ${ }^{26}$ Ceci se traduit aussi par la différentiation des programmes sociaux, comme l'a expliqué notamment Berkowitz: l'attaque contre le welfare, programmes bénéficiant majoritairement aux minorités pauvres, va de pair avec la défense de programmes d'assurance sociale, tels que la Social Security, perçus comme programmes 'mérités' par les cotisations et bénéficiant à la classe moyenne blanche. ${ }^{27}$ Cette perception raciale des programmes sociaux a été notamment mise en lumière par Robert Lieberman. ${ }^{28}$ Une telle attaque s'est construite autour de la notion de responsabilité personnelle, 
véritable mot code pour jouer sur les préjugés raciaux et ainsi attaquer les politiques sociales. ${ }^{29}$ C'est notamment le refus total des libéraux et du Parti démocrate de discuter des problèmes réels que rencontre la underclass noire qui a permis aux conservateurs et au Parti républicain de dominer le discours pendant longtemps. ${ }^{30}$

Le discours républicain bâti autour de la notion de responsabilité personnelle a émergé à la fin des années 1970 et au début des années 1980 dans un contexte économique et social particulier. La convergence entre une situation économique en déclin et l'émergence de la underclass (principalement noire et hispanique) a permis de raviver la vieille théorie de la perversion. Certains, comme l'historien Michael B. Katz, appellent cela le concept du danger moral. Selon cette théorie, les politiques sociales favorisent la paresse, l'oisiveté et l'immoralité. Cette théorie, largement en circulation dans le monde anglo-américain depuis le $16^{\text {ème }}$ siècle et la législation anglaise sur la pauvreté (Poor Law), défend aussi l'idée selon laquelle refuser des subventions et de l'aide, et de ce fait forcer les gens au travail, revient à leur rendre service, puisque cela les obligerait à être responsables. ${ }^{31}$ Durant les années 1980 , cette théorie a été largement diffusée auprès du public grâce à l'ouvrage Losing Ground de Charles Murray, un politologue conservateur et libertaire, publié en $1984 .{ }^{32}$ Cette théorie a pu être ravivée car la fin des années 1970 a été un moment où les attitudes des Blancs concernant les Noirs avaient changé de façon assez marquée par rapport au début des années 1960. Les émeutes raciales durant les années 1960 et les mouvements nationalistes ont contribué à donner une image plus négative de la communauté noire. ${ }^{33}$ De plus, dans la population blanche, il existe l'idée assez répandue que le Civil Rights Act de 1964 a mis fin à toute discrimination. ${ }^{34}$ Cette tendance s'est encore accrue aujourd'hui. Si en 1977 encore $40 \%$ de la population blanche citait la discrimination comme cause principale d'inégalités raciales, ce chiffre est tombé a $30 \%$ en 2008. ${ }^{35}$ Eduardo Bonilla-Silva constate que les attitudes de la population blanche concernant les questions raciales ont peu changé depuis les années $1980 .^{36}$ De façon assez ironique, une part croissante de la population noire partage cette opinion et mentionne de plus en plus un manque de volonté et d'efforts comme cause d'inégalités. ${ }^{37}$

Bien que le président Richard Nixon ait déjà commencé à tenir un discours sécuritaire durant son premier mandat, l'atmosphère générale était encore bien trop libérale pour attaquer les politiques sociales de front. Bien au contraire, Nixon fut l'un des présidents les plus actifs dans l'expansion des politiques sociales en essayant de battre les Démocrates à leur propre jeu. ${ }^{38}$ Le tournant réel est arrivé en 1978 avec la révolte des impôts (La Tax Revolt a démarré en Californie avec le débat autour des impôts sur l'immobilier et la propriété), indiquant un clair refus de la part des classes ouvrières et moyennes blanches de continuer à payer pour des politiques sociales jugées inefficaces. ${ }^{39}$ C'est seulement avec la candidature, puis avec la présidence, de Ronald Reagan que le discours prônant la responsabilité personnelle comme solution miracle aux déviances causées par les politiques «irresponsables » des Démocrates a vraiment eu du succès auprès des électeurs, et particulièrement des Reagan-Democrats. ${ }^{40}$ Selon Lieberman, ce discours diviseur qui joue sur la question raciale a contribué à fausser l'image que se fait la population des politiques sociales et notamment à minimiser le soutien exprimé pour les politiques sociales ${ }^{41}$ Lorsque les programmes sont critiqués de manière générale en termes racialisés (welfare), l'opinion publique demande des mesures plus dures. Lorsque des domaines sont mentionnés, tels que la santé ou l'éducation, l'opinion publique a tendance à favoriser les dépenses de l'Etat. ${ }^{42}$ 
11 L'idéologie reaganienne de la responsabilité personnelle se construit autour de trois axes.

12 Premièrement, Reagan avait une forte admiration pour les self-made men, ces personnes ayant gravi l'échelle sociale à force de travail acharné et de persistance. Certains documents conservés dans les archives présidentielles attestent que Reagan était persuadé que toutes les barrières de la discrimination avaient été abattues, que les questions raciales ne jouaient plus aucun rôle. Selon lui, les Africains-Américains et les Hispaniques ne souffraient plus d'aucune limite à l'intégration et avaient de ce fait les mêmes possibilités de réussite que la population blanche. ${ }^{43}$ Il croyait de plus fermement que la politique économique de l'offre était la solution pour l'économie des Etats-Unis. Afin de rester compétitif et de stimuler l'investissement, une baisse des réglementations gouvernementales était nécessaire, ainsi que la diminution des impôts pour les riches. Dans cette conception, partagée par la Première Ministre britannique Margaret Thatcher, les gains faits par les couches supérieures de la population finiraient tôt ou tard par bénéficier à la population entière. Selon Bruce Jansson, le monde idéal de Reagan ressemble au Gilded Age des Etats-Unis du $19^{\text {ème }}$ siècle, une période de déréglementation totale avec un fort accent sur l'individualisme. ${ }^{44} \mathrm{C}^{\text {'est }}$ notamment Katz qui analyse le discours conservateur prônant les méfaits de l'aide sociale et vantant les mérites de la charité privée comme une interprétation mythique de la fin du $19^{\text {ème }}$ siècle..$^{45}$ Ces idées ont permis de construire un discours présentant les politiques sociales interventionnistes des Démocrates comme irresponsables, en les désignant comme la cause non-seulement de la crise économique, mais aussi de la "dépravation » de la underclass. Le Parti républicain, au contraire, proposait d'opérer des coupes dans les budgets des programmes sociaux, afin de sauver l'économie, mais aussi de responsabiliser les bénéficiaires de subventions sociales en les mettant au travail. De plus, la vision du monde reaganienne et conservatrice permettait aussi de rendre les pauvres responsables de leur pauvreté, puisque, selon les Républicains, toutes les conditions étaient remplies pour devenir riche si on le voulait et si on travaillait dur. ${ }^{46}$ Le contraste est flagrant: le programme du Parti démocrate de la même année mettait l'accent sur la responsabilité de l'Etat envers les citoyens et l'aide aux populations ayant souffert de discrimination. ${ }^{47}$

13 Cette construction idéologique autour de l'idée de responsabilité personnelle peut se voir dans le discours sur l'Etat de l'Union tenu par Reagan en 1986.

We do not face large deficits because American families are undertaxed; we face those deficits because the federal government overspends.

The detailed budget that we will submit will meet the Gramm-Rudman-Hollings target for deficit reductions, meet our commitment to ensure a strong national defense, meet our commitment to protect Social Security and the truly less fortunate, and, yes, meet our commitment to not raise taxes. How should we accomplish this? Well, not by taking from those in need. As families take care of their own, government must provide shelter and nourishment for those who cannot provide for themselves. But we must revise or replace programs enacted in the name of compassion that degrade the moral worth of work, encourage family breakups, and drive entire communities into a bleak and heartless dependency. ${ }^{48}$

14 Ce court extrait montre bien comment les différents éléments sont intrinsèquement liés : au premier plan vient le souci de l'équilibre budgétaire et de la dette, tout en insistant sur le soutien de certains programmes et postes budgétaires, tel que l'armée. 
Il faut remarquer que Reagan se positionne comme étant en faveur de certains programmes sociaux, citant ici la Social Security (programme de retraites). La Social Security est un des programmes les plus populaires aux Etats-Unis, très difficile à attaquer, et qui jouit d'un fort soutien dans la classe moyenne blanche. ${ }^{49}$ Toutefois cette défense de la Social Security est immédiatement suivie par un point beaucoup plus central du programme reaganien: la promesse de ne pas augmenter les impôts. Par cette association, il crée une situation de pénurie, des ressources limitées en matière de programmes sociaux, et donc un besoin de différencier entre ceux qui "méritent " vraiment d'en bénéficier, et ceux qui finalement sont des "undeserving poor», des pauvres qui, eux, par leur manque de valeurs, d'efforts, et de morale, ne "méritent " pas de bénéficier de programmes sociaux. Par les références à l'éthique de travail, aux familles divorcées, et à des communautés entières dans la dépendance, l'association se fait avec la underclass noire. ${ }^{50}$

15 Le discours de Reagan lie particulièrement la question de responsabilité à celle des impôts. Dans la conception reaganienne, les Démocrates veulent trop dépenser, prenant à ceux qui travaillent dur, pour donner à ceux qui ne le méritent pas, et empêchent la croissance économique à cause de réglementations excessives. Ces deux idées sont regroupées sous le terme «big government » qui représente à lui seul toute l'irresponsabilité du Parti démocrate. Le "big government » diabolisé qui opprime le petit travailleur blanc honnête est une idée que Reagan avait empruntée notamment à George Wallace, un Démocrate sudiste qui avait axé sa campagne présidentielle en 1964 autour d'une rhétorique de populisme racial..$^{51}$ Elle permet en même temps de dénoncer trop d'impôts et trop de dépenses sociales pour des bénéficiaires représentés comme « irresponsables et fainéants ${ }^{52} \mathrm{Si}$ les attaques directes contre les politiques sociales sont assez rares, les attaques contre le "big government " sont un thème obligatoire dans les discours de Reagan et sont le mieux illustrées par sa célèbre citation: "Government is not a solution to our problem government is the problem. " ${ }^{53}$

Le discours jouant de la notion de responsabilité mis en place par Reagan s'est montré particulièrement durable. En effet, George H.W. Bush a articulé sa candidature en 1988 autour du thème de l'irresponsabilité des politiques Démocrates, notamment à travers son spot publicitaire de campagne des « revolving doors ». Ce spot dénonçait les positions des Démocrates en faveur des droits des prisonniers, prétendant que cela favorisait la criminalité. Ce spot a été largement interprété comme étant du populisme racial, ce que la campagne de Bush a nié. ${ }^{54}$ Bien que Bush ait caressé l'idée d'intervenir dans les politiques sociales, il a consacré peu de moyens et d'attention à ses projets sur l'éducation, les programmes de lutte contre la toxicomanie, et même pour les programmes sociaux. L'idée conservatrice fondamentale était maintenue: « you can't solve problems by throwing money at them. $\|^{55}$

$17 \mathrm{Vu}$ la prégnance de ce cadre rhétorique conservateur, au bout de douze ans de présidence républicaine, en 1992, certains analystes politiques tels que le journaliste politique et chercheur Thomas B. Edsall en étaient venus à la conclusion que les questions du travail, de l'autonomie, et de l'indépendance (self-reliance) qui se trouvent distillées dans la notion de responsabilité personnelle, étaient un facteur que le Parti démocrate ne pouvait se permettre d'ignorer s'il voulait retrouver une majorité présidentielle. ${ }^{56}$ 


\section{Bill Clinton et G.W. Bush : deux approches hybrides}

Bill Clinton partageait ardemment cet avis, soulignant le tournant conservateur qu'avait pris l'électorat, ainsi que la résonance du discours sur la responsabilité personnelle. Déjà lors de la défaite de George McGovern en 1972, Bill Clinton avait fait le constat que le Parti démocrate n'était plus en phase avec ses électeurs traditionnels. Clinton dit être devenu un New Democrat cette année-là, du moins du point de vue idéologique, puisque le terme a été créé ultérieurement. Plus tard, il cite la responsabilité come élément fondamental dans le renouveau idéologique des New Democrats, le retour au valeurs fondamentales du Parti démocrate selon lui : leur nouvelle troisième voie était fondée sur le bon sens et sur une dévotion commune aux deux plus anciennes valeurs du Parti, à savoir l'égalité des chances et la responsabilité. 57

De plus, il avait vu le tort qu'avait selon lui causé l'attitude libérale jusqu'alors. Cette attitude consistait tout simplement à refuser de parler des problèmes de la underclass. Selon Al From, fondateur du Democratic Leadership Council et conseiller politique de Bill Clinton, ce dernier insistait sur le besoin d'un renouveau intellectuel et politique du parti..$^{58}$ Clinton prit soin en 1992 de ne pas apparaitre trop libéral. Ceci faisait partie de la volonté de donner une nouvelle image au Parti démocrate, de montrer des New Democrats responsables qui s'opposent aux politiques sociales dépensières, qui sont en faveur d'un équilibre budgétaire et qui veulent limiter l'envergure et la portée de l'Etat. Cette nouvelle volonté des New Democrats était exprimée dans le slogan de campagne « to end welfare as we know it ${ }^{59}$ Clinton a en effet décidé de jouer sur le terrain des Républicains, allant jusqu'à se réclamer des mêmes valeurs conservatrices que le Parti républicain ${ }^{60}$ dans son discours sur l'Etat de l'Union de 1993 : «I believe we will find our new direction in the basic old values that brought us here over the last two centuries : a commitment to opportunity, to individual responsibility, to community, to work, to family, and to faith. $»^{61}$

20 Certes, les valeurs comme l'égalité des chances, le travail, la famille et la foi font partie des bases communes de tous les Américains. ${ }^{62}$ Toutefois, ici, c'est le contexte qui permet un rapprochement avec une interprétation plus conservatrice des valeurs énumérées par Clinton. Ce dernier avait mené sa campagne électorale de 1992 en mettant en avant son affiliation modérée et en se montrant résolument New Democrat. En effet, les deux notions de famille et de travail sont utilisées dans la construction conservatrice comme des éléments venant compléter le discours autour de la notion de responsabilité personnelle, mettant l'accent sur plus de travail et plus de soutien familial par opposition à plus d'aide de l'Etat. Laura Katz Olson, professeur de sciences politiques, considère le familisme (c'est-à-dire la primauté de la famille), la responsabilité personnelle et un plus grand individualisme, comme les trois principes de l'antiétatisme des conservateurs avancé par le Parti républicain. ${ }^{63}$

21 L'autre facette des politiques sociales, la politique fiscale, a été un succès, et Clinton a non seulement réussi à combler le déficit budgétaire largement aggravé par Reagan et Bush, mais à produire un excédent budgétaire en 1998. Cependant, concernant les politiques sociales, Clinton s'est fait happer par sa stratégie centriste, voire conservatrice. Sa réforme de la santé avec couverture universelle a été torpillée par les Républicains..$^{64}$ Theda Skocpol fait remarquer que la réforme de la santé de Clinton, dans sa construction, prenait bien en compte la rhétorique républicaine, dans la 
mesure où il avait pris soin de ne pas devoir lever de nouveaux impôts pour financer celle-ci et qu'il s'appuyait sur le modèle existant, régulant la compétition de marché, plutôt que de créer une couverture santé publique. Toutefois ceci a produit un enchevêtrement bureaucratique que le public a rejeté. Ce labyrinthe bureaucratique s'est avéré vulnérable à une attaque sur le thème du "big government ", la bureaucratie représentant ce qu'il y a de plus négatif dans la doxa conservatrice ${ }^{65}$ Grâce à la pression des élections présidentielles à venir, les Républicains ont réussi à pousser Clinton à signer une réforme des programmes sociaux très conservatrice: le Personal Responsibility and Work Opportunity Act de 1996. De nombreuses clauses mettaient l'accent sur des mesures de responsabilisation (selon la conception républicaine et conservatrice) : limitation de la durée des prestations, restriction des critères d'accès aux droits, limitations sévères d'accès aux droits pour les parents adolescents, et de fortes exigences et incitations au travail pour les bénéficiaires de TANF (Temporary Assistance for Needy Families). Par ailleurs, le programme TANF (auparavant nommé le AFDC [Assistance to Families with Dependent Children], un des plus gros programmes d'aide sociale) allait dorénavant être limité à un maximum de 5 années par foyer, mettant fin à la conception qui existait auparavant, selon laquelle cette aide était un droit pour les personnes démunies ${ }^{66}$ Certaines mesures de TANF visaient à accroitre la responsabilité parentale, comme par exemple les clauses stipulant la perte de droits pour des bénéficiaires (mariés ou non) qui auraient d'autres enfants durant la période où ils reçoivent des aides. Certains critiques, tels que David Scmidtz et Robert Goodin, considèrent qu'avec la signature du Personal Responsibility and Work Opportunity Act Clinton a achevé la révolution reaganienne. ${ }^{67}$

22 Si Clinton a décidé de jouer sur le terrain conservateur jusque là associé aux Républicains, l'approche de G.W. Bush entre 2000 et 2008 a aussi un certain caractère hybride, bien que nettement moins marqué.

G.W. Bush était bien décidé à éviter les erreurs commises par son père ayant coûté sa réélection à ce dernier. Bush s'est vu comme l'héritier politique de Reagan et a déclaré ouvertement vouloir poursuivre ses politiques. En revanche, il a fait attention à paraître moins dur en se penchant sur la réforme de l'éducation (No Child Left Behind Act de 2001) et il s'est présenté comme un « compassionate conservative. » Ceci lui a permis de s'attirer le soutien des modérés pendant sa campagne de 2000 et au début de sa présidence. ${ }^{68}$ Le choix de l'éducation comme domaine social pour exprimer son conservatisme de compassion s'est basé sur deux critères. L'éducation fait partie des domaines pour lesquels les électeurs sont prêts à accepter plus de dépenses. ${ }^{69}$ De plus, l'éducation tombe en priorité sous la responsabilité des Etats, l'intervention de l'Etat fédéral étant assez limitée. Par cet aspect, la réforme de l'éducation reste compatible avec la critique conservatrice du «big government ». G. W. Bush a aussi mis ce point en avant dans les programmes de 2000 et de 2004. ${ }^{70}$ On peut qualifier NCLB de réforme compatissante car elle met, par exemple, l'accent sur des programmes d'aide à l'apprentissage pour les enfants pauvres et sur la diminution de la fracture scolaire autant au niveau racial qu'au niveau des classes sociales. ${ }^{71}$ En revanche, parmi les plus grands manquements de cette réforme, on peut citer les problèmes budgétaires. En effet, entre l'année fiscale 2002 et l'année fiscale 2008, l'écart entre les fonds alloués et les fonds effectivement versés s'est élevé à 70,9 milliards de dollars. Cet écart est passé à 85,7 milliards de dollars en 2009.72 C'est Barack obama qui a remédié à ce non-versement de fonds en 2010. En 
septembre de cette année la totalité des 97,4 milliards des dollars alloués par le American Recovery and Reinvestment Act de 2009 a été versée,73 comblant ainsi le trou laissé par l'administration Bush. penser, ni la notion de responsabilité personnelle, ni la critique d'une trop grande intervention de l'État ne sont des questions étrangères à la communauté noire. En effet, les notions de "personal responsibility " et de "self-help» font partie intégrante d'une tradition intellectuelle noire portée par des personnalités aussi diverses que Booker T. Washington, W.E.B. Du Bois, ou plus récemment par Barbara Jordan. ${ }^{77}$ Les mêmes thèmes ont été développés et discutés depuis la fin du XIXème siècle pour étayer des thèses parfois opposées, soit intégrationnistes (Du Bois et Jordan, par exemple), soit 
séparatistes : la responsabilité personnelle et le "self-help» exprimaient la déception face à la détérioration des relations raciales et le manque d'espoir concernant la possibilité d'une société mixte. ${ }^{78} \mathrm{La}$ Nation of Islam et le Black Panthers Party ont poussé cette idée à l'extrême en prônant la création d'une nation noire séparée. ${ }^{79}$ Obama s'inscrit donc dans une longue lignée de penseurs noirs qui appellent à plus de responsabilité personnelle.

La critique de l'interventionnisme étatique se retrouve aussi dans la communauté noire. L'un des plus fervents critiques noirs du «big government » est le juge de la Cour Suprême Clarence Thomas. Bien que soit un fervent conservateur, son opposition à l'intervention de l'État est tout de même racialement motivée. Selon lui, la dépendance aux aides de l'État est dégradante et infantilisante. Elle met les Noirs dans une situation de quasi-tutelle de l'État et les laisse exposés à l'intrusion de l'État dans leur vie privée. Pour Clarence Thomas, dont la vision a été profondément influencée par son grandpère, cette situation est analogue à ce que la communauté à subi durant l'esclavage ou la ségrégation. Cette vision est donc très différente de la vision classique du désengagement de l'État au détriment de la communauté noire, mais elle reflète plutôt un désir d'affirmation de liberté et d'indépendance..$^{80}$

Bien que l'utilisation de la notion de responsabilité personnelle existe dans la tradition rhétorique noire, le transfert de celle-ci dans le discours d'un chef d'Etat démocrate n'est pas pour autant facile. L'échec de Clinton a montré qu'il n'est pas possible pour un président démocrate d'utiliser tel quel le discours conservateur dans l'espoir de le retourner à son avantage. L'approche de George W. Bush a aussi montré que les Démocrates n'ont pas le monopole de la réforme sociale positive, bien que Bush se soit cantonné à un domaine assez compatible avec l'idéologie conservatrice et l'image du Parti républicain.

31 Avec la présidence de Barack Obama, le Parti démocrate est entré dans une nouvelle période en ce qui concerne l'utilisation de la notion de responsabilité. Obama est absolument conscient de l'importance du discours sur les valeurs, telles que la responsabilité, et de son utilisation politique. ${ }^{81}$ Certains critiques lui ont d'ailleurs reproché de parler uniquement de responsabilité personnelle, en laissant de côté le rôle de l'Etat dans la lutte contre les inégalités. ${ }^{82}$ Une analyse détaillée de son discours révèle que les idées d'Obama sont plus nuancées, qu'il construit un message complexe alliant responsabilité personnelle et responsabilité de l'Etat. Ce discours nuancé et enchevêtré représente une évolution plus marquée dans le discours politique noir de ces dernières décennies, qui met en avant justement cette voie centriste entre le discours du «tout-Etat » libéral traditionnel et le discours du «tout-individuel » des conservateurs. ${ }^{83}$

32 Alors que dans le discours de Reagan, la notion de responsabilité n'est pratiquement jamais mentionnée, le terme abonde dans le discours démocrate. Chez Reagan la responsabilité transpire seulement dans le discours général, par sous-entendu. La responsabilité personnelle n'est pratiquement jamais mentionnée mot pour mot. En revanche, chez les Démocrates le mot "responsabilité » revient continuellement. Ceci est certainement motivé en partie par le souhait de contrer les accusations d'irresponsabilité. Dans le cas d'Obama il s'agit aussi de mettre en place une nouvelle utilisation du terme. Dans ses discours, Obama embrasse le discours conservateur comme d'autres Africain-Américains avant lui sur la notion de responsabilité. La grande différence est qu'Obama construit un discours qui utilise la notion de 
responsabilité afin de promouvoir l'expansion de politiques sociales, ainsi qu'une plus grande intervention et participation de l'Etat fédéral dans les politiques sociales. Par cette approche, Obama se pose en contraste net, non seulement avec Républicains, mais aussi avec les New Democrats. Obama développe la notion de responsabilité à différents niveaux et l'applique à différents domaines des politiques sociales, notamment l'éducation, la santé, et le travail.

Dans une certaine mesure, Obama s'aventure aussi sur le terrain conservateur et républicain, tout comme l'avait fait Clinton. Obama est tout à fait conscient que le discours de responsabilité personnelle tenu par les Républicains a toujours une forte résonance dans l'électorat. En ce qui concerne l'éducation notamment, Obama insiste fréquemment sur la responsabilité qu'ont les élèves concernant leur scolarité : «And that's what I want to focus on today: the responsibility each of you has for your education. I want to start with the responsibility you have to yourself. $»^{84}$ Le sujet de la responsabilité personnelle reste sensible, justement à cause de la connotation péjorative et accusatrice que le terme a pris dans l'usage qu'en font les Républicains. Obama a aussi été confronté à cela et a essayé de dissiper les accusations de racisme.

We also know that as significant as these reforms are, there's going to be one more ingredient to really make a difference: parents are going to have to get more involved in their children's education. [...] Then some people say, well, why are you always talking about parental responsibility in front of black folks? And I say, I talk about parent responsibility wherever I talk about education. ${ }^{85}$

La notion de responsabilité personnelle se retrouve aussi dans les politiques de Barack Obama, particulièrement en ce qui concerne la santé. Dans la réforme de la santé qu'il a introduite en 2009 et qui a été votée en 2010 sous le nom de Patient Protection and Affordable Care Act, la responsabilité personnelle se trouve en toutes lettres, dans le paragraphe intitulé «Individual Responsibility» qui expose le «individual mandate.» Le mandat individuel est l'obligation pour tout citoyen de contracter une assurance maladie sous peine d'amende. La possibilité de faire preuve de responsabilité personnelle est aussi donnée dans cette nouvelle législation par le biais des soins préventifs qui sont désormais couverts. Ceci permet aux individus de mieux prendre soin d'eux-mêmes et d'être une charge moins lourde pour le système de santé. ${ }^{86}$

Toutefois, toujours dans le domaine de la santé, Obama ne s'arrête pas à une simple responsabilité personnelle. Exprimée à travers le mandat individuel, la notion de responsabilité se retrouve cependant dans la partie de la législation intitulée «Shared Responsiblility $»{ }^{87} \mathrm{La}$ responsabilité personnelle ici ne sert pas de prétexte pour désengager la collectivité, mais bien au contraire à construire un système solidaire pour le bénéfice de tous. En effet, le système d'assurance maladie ne fonctionne que si tout le monde participe en contractant une assurance. La responsabilité dans la santé est échelonnée entre les différents acteurs sociaux. En effet, la deuxième partie de la section "Shared Responsibility", intitulée "Employer Responsibility", prévoit aussi le partage de la responsabilité avec les employeurs. Le Affordable Care Act oblige, sous peine d'amende, les entreprises de plus de 50 salariés à temps plein à proposer une couverture santé minimale définie par la réforme. ${ }^{88}$

36 Cette idée de responsabilité partagée et échelonnée entre les différents acteurs est également appliquée par Obama au domaine de l'éducation. Obama prend soin de montrer qu'il ne considère pas que seule la responsabilité personnelle compte, mais 
bien que la responsabilité engage tous les niveaux de la nation, depuis l'Etat fédéral jusqu'à l'élève, en passant par toutes les étapes intermédiaires :

So, yes, our federal government has responsibilities that it has to meet, and I will keep on making sure the federal government meets those responsibilities. Our governors, our superintendants, our states, our school districts have responsibilities to meet. And parents have responsibilities that they have to meet. And our children have responsibilities that they have to meet. ${ }^{89}$

Obama utilise donc la notion de responsabilité pour mettre en avant plus d'intervention étatique, y compris dans le domaine de l'éducation (constitutionnellement une prérogative des Etats), ce qui le place ainsi en fort contraste avec les Républicains. Par cette stratégie Obama parvient à lier les approches conservatrice et libérale, à réunir l'ancien discours des Démocrates et celui des Républicains : tout le monde a son niveau de responsabilité. Pour lui il s'agit là d'une responsabilité mutuelle, réciproque, de responsabilités indissociables. Il ne peut donc en aucun cas s'agir uniquement de responsabilité personnelle comme c'est le cas dans l'interprétation conservatrice de la notion revendiquée par le Parti républicain. En cela il se réclame clairement de l'idéologie démocrate:

That is one of the things that makes me a Democrat, I suppose - this idea that our communal values, our sense of mutual responsibility and social solidarity, should express themselves not just in the church or mosque or the synagogue; not just on the blocks where we live, in the places where we work, or within our own families; but also through our government. ${ }^{90}$

Pour Obama c'est donc clairement une responsabilité étatique qui doit répondre à cette exigence de responsabilité personnelle, il n'est pas question de s'en remettre aux œuvres de charité, forme de désengagement de l'Etat.

Cette plus grande implication étatique touche aussi le domaine du travail. En effet, si Obama considère que les individus doivent faire des efforts, il n'en est pas moins persuadé que c'est l'Etat qui doit leur en fournir les moyens, par exemple en offrant des possibilités de formation aux individus en recherche d'emploi. ${ }^{91}$ Il s'oppose en cela par exemple au Personal Responsiblity and Work Opportunity Act de 1996 qui certes demande une participation à travers le travail, mais n'offre pas de formations. ${ }^{92}$

Cette conviction d'une plus grande responsabilité gouvernementale se trouve appliquée concrètement dans la réforme de la santé. En effet, plusieurs dispositions de la nouvelle législation élargissent le rôle joué par l'Etat dans le domaine de la santé. Désormais l'Etat intervient en réglementant le contenu des contrats d'assurance maladie offerts par les assureurs privés. De plus, la nouvelle législation autorise les Etats à proposer des assurances maladie minimum pour les personnes à faibles revenus n'ayant pas droit à Medicaid, l'assurance maladie publique pour les pauvres. De plus, la législation prévoit l'élargissement des droits de Medicaid pour inclure une plus grande partie de la population à faible revenus. ${ }^{93}$ Un autre point central de la réforme Obama sont les subventions de l'Etat pour aider les personnes non-éligibles pour Medicaid, mais ayant des revenus relativement modestes, à souscrire une assurance maladie. ${ }^{94} \mathrm{C}^{\prime}$ est donc l'un des aspects révélant nettement l'influence de l'idéologie libérale, dans le sens où ces subventions sont une aide directe de l'Etat pour l'individu qui ne peut pas se prendre en charge seul. De plus, les bénéficiaires sont ici des personnes considérées comme capables de se prendre en charge, et non pas de personnes tombant sous la définition de la dépendance communément acceptée comme naturelle. ${ }^{95}$ 
41 Obama travaille donc la notion de responsabilité à plusieurs niveaux: individuel, collectif, et étatique. La responsabilité collective inclut l'idée de responsabilité partagée et la responsabilité étatique concerne autant les Etats que l'Etat fédéral. Mais la force rhétorique d'obama réside dans le fait qu'il insère les multiples déclinaisons de la responsabilité dans la notion plus large de responsabilité réciproque. Déjà en 1992, Thomas Edsall avait relevé que le public ne s'intéressait pas aux des responsabilités d'accompagnement (accompanying responsibilities) ou des obligations réciproques (reciprocal obligations), c'est-à-dire à l'idée que l'Etat a des devoirs envers ses citoyens. Selon Edsall, l'échec des Démocrates qui n'ont su introduire aucune de ces notions dans le débat sur les politiques sociales a ouvert la porte aux conservateurs pour dominer le discours. ${ }^{96}$ En revanche, dans le discours d'Obama se dessine un va-et-vient entre les différents niveaux de responsabilité, et il insiste sur le fait que l'un ne peut pas fonctionner sans l'autre: on ne peut pas demander aux citoyens de prendre leurs responsabilités si l'Etat se désengage. En cela le discours d'Obama reflète l'opinion publique concernant les politiques sociales, à savoir une combinaison des deux grandes tendances: l'importance de la responsabilité étatique dans la prise en charge des personnes en difficulté, et une demande morale de plus de responsabilité personnelle afin de compléter cet effort fait par l'Etat. ${ }^{97}$

Obama adopte ce discours, par exemple, en matière d'éducation, où il met en avant le fait que les efforts faits par l'Etat ne sont rien sans la participation des élèves : «I'm working hard to fix up your classrooms and get you the books, equipment and computers you need to learn. But you've got to do your part too. ${ }^{98}$ Obama insiste en effet sur le fait que chacun doit assumer sa part de responsabilité afin que les choses se fassent :

But to paraphrase Dr. King, education isn't an either/or proposition. It's a both/and proposition. It will take both more focus from our parents, and better schooling. It will take both more money, and more reform. It will take both a collective commitment, and a personal commitment. ${ }^{99}$

C'est grâce à cette responsabilité réciproque qu'Obama parvient à construire un nouveau discours cohérent utilisant toutes les facettes de la notion de responsabilité. En rendant la responsabilité personnelle et la responsabilité gouvernementale interdépendantes, Obama parvient à légitimer une plus grande intervention gouvernementale, sans pour autant apparaitre comme un Démocrate traditionnel qui chercherait à compenser les manquements personnels des individus par des charges injustement imposées à la collectivité.

En résumé, depuis les années 1980, les conservateurs ont construit un discours autour de la responsabilité personnelle dans le but de justifier le désengagement de l'Etat fédéral dans les politiques sociales. La réappropriation de la notion de responsabilité personnelle par les New Democrats durant la présidence de Bill Clinton a certes été un succès électoral, mais elle a eu des effets contraires à ceux souhaités en ce qui concerne les politiques sociales. C'est Obama qui a su transférer la notion de responsabilité personnelle avec succès dans son discours, en élargissant le champ de la notion de responsabilité à tous les acteurs sociaux et politiques, et surtout en construisant son discours autour de la notion de responsabilité réciproque, engageant à parts égales les différents acteurs, dépendants les uns des autres, et complémentaires. Dans le Affordable Care Act, on peut clairement identifier le lien entre rhétorique et politique, puisque la législation reflète le discours. En effet, le texte associe la responsabilité 
personnelle exprimée, par exemple, par le mandat individuel, à la responsabilité étatique d'aider les personnes à assumer cette responsabilité par le biais des subventions. Si on peut constater un certain succès de la rhétorique dans le vote même de la réforme, il faut aussi admettre que celle-ci a eu un impact limité : la public option (l'option publique: la possibilité de souscrire une assurance maladie proposée par l'Etat) a été écartée très tôt durant le processus législatif, limitant ainsi l'implication de l'Etat. L'extension de Medicaid, et donc une plus grande prise en charge des populations pauvres par l'Etat, a été rendue facultative pour les Etats après le procès Sebelius. ${ }^{100}$ Ces aspects montrent que les conservateurs ont encore une influence certaine et parviennent à influer sur les choix législatifs.

\section{BIBLIOGRAPHIE}

111th Congress of the United States. "Patient Protection and Affordable Care Act of 2010." Government Printing Office. Consulted 26 Apr. 2013. <http://www.gpo.gov/fdsys/pkg/ BILLS-111hr3590enr/pdf/BILLS-111hr3590enr.pdf>.

Akerlof, George A., Janet L. Yellen and Michael L. Katz. "An Analysis of Out-of-Wedlock Childbearing in the United States," The Quarterly Journal of Economics 111:2 (1996): 277-317.

Back, Les and John Solomons, eds. Theories of Race and Racism: A Reader. $2^{\text {nd }}$ ed. London: Routledge, 2009.

Berkowitz, Edward D. America's Welfare State: From Roosevelt to Reagan. Baltimore: Johns Hopkins University Press, 1991.

Black Panther Party Platform 1966. Columbia American History Online. Consulted 12. Dec. 2014. <http://caho-test.cc.columbia.edu/ps/10127.html>.

"Blacks Upbeat about Black Progress, Prospects: A Year After Obama's Election." Pew Research Center. January 21, 2010. <http://www.pewsocialtrends.org/2010/01/12/blacks-upbeat-aboutblack-progress-prospects/ >.

Bobo, Lawrence D. "Somewhere between Jim Crow and Post-Racialism : Reflections on the Racial Divide in America Today." Daedalus $140: 2$ (2011) :11-36.

Bonilla-Silva, Eduardo. Racism without Racists : Colorblind Racism and the Persistence of Racial Inequality in America. Lanham, Md : Rowman and Littlefield, 2014.

Brewer, Mark D. and Jeffrey M. Stonecash. Split: Class and Cultural Divides in American Politics. Washington: CQ Press, 2007.

Bush, George H. W. "Inaugural Address," January 20, 1989. Online by Gerhard Peters and John T. Woolley, The American Presidency Project. <http://www.presidency.ucsb.edu/ws/?pid=16610 >.

Bush, George W. "State of the Union Address." 27 January 2001. Consulted 12. Dec. 2013. Online by Gerhard Peters and John T. Woolley, The American Presidency Project. <http://www.let.rug.nl/ usa/presidents/george-w-bush/state-of-the-union-2001.php >. 
Clinton, William J. "Address Before a Joint Session of Congress on Administration Goals," February 17, 1993. Online by Gerhard Peters and John T. Woolley, The American Presidency Project. Consulted 12. Dec. 2013. <http://www.presidency.ucsb.edu/ws/?pid=47232>.

Coste, Françoise. « La longue marche de Ronald Reagan contre le mouvement des droits civiques : une croisade idéologique finalement vaine ? » Communication, journée d'études « Mémoires du mouvement pour les droits civiques ». Université Toulouse-Le Mirail, 28 février 2014.

Coste, Françoise. Reagan. Paris, France : Perrin, 2015.

Coste, Françoise. "Ronald Reagan and a New Partisan Identity: The Case of the Reagan Democrats." Communication, Colloque "American Identities : In Relation and Interaction », Université Toulouse-Le Mirail, 10-11 Juin 2010.

Cunnigen, Donald and Marino A. Bruce, eds. Race in the Age of Obama. Research in Race and Ethnic Relations. vol. 16. Bingley, UK: Emerald, 2010.

Du Bois, W.E.B. The Souls of Black Folk. ed. Jim Manis, 2006. Electronic Classic Series. Hazelton, PA: PSU, 2014

Edsall Thomas B. and Mary D. Edsall. Chain Reaction: The Impact of Race, Rights, and Taxes on American Politics. New York: Norton and Company, 1992.

Ed.gov. Recovery Act. U.S. Department of Education. N.d. Consulted. 16 Feb. 2011. http:// www.ed.gov/recovery>.

Ed.gov. The No Child Left Behind Act of 2001. U.S. Department of Education. 7 Jan. 2002. Web. 10 Oct. 2010. http://www2.ed.gov/nclb/overview/intro/execsumm.pdf>.

Emerson, Ralph Waldo. The Conduct of Life. 1866. Boston: Ticknor and Fields, 1868.

Feagin, Joe, R. White Party, White Government: Race, Class, and U.S. Politics. New York: Routledge, 2012.

From, Al. The New Democrats and the Return to Power. New York: Palgrave Macmillan, 2013.

Gerring, John. Party Ideologies in America: 1828 - 1996. Cambridge: Cambridge University Press, 1998.

Gilens, Martin. Why Americans Hate Welfare: Race, Media, and the Politics of Antipoverty Policy.

Chicago: University of Chicago Press, 1999.

Goodin, Robert E., ed. The Oxford Handbook of Political Science. 2009. New York: Oxford University Press, 2011.

Harwood, John. "Don't Dare Call the Health Law 'Redistribution'," The New York Times, 23 Nov. 2013. Consulted 21 Apr. 2015 <http://www.nytimes.com/2013/11/24/us/dont-dare-call-thehealth-law-redistribution.html>.

History, Art, and Archives: United States House of Representatives. Barbara Jordan. Consulted 12. Dec. 2014. <http://history.house.gov/People/Listing/J/JORDAN,-Barbara-Charline$\% 28 \mathrm{~J} 000266 \% 29 />$.

Herakova, Liliana L., Dijana Jelača, razvan Sibii, and Leda Cooks. "Voicing Silence and Imagining Citizenship : Dialogues about Race and Whiteness in a "Postracial" Era." Communication Studies $62: 4$ (2011) : 372-88.

Hermer, Laura D. "Personal Responsibility: A Plausible Social Goal, but Not for Medicaid Reform," The Hastings Center Report 38:3 (2008): 16-19. 
Hochschild, Jennifer L. Facing Up to the American Dream: Race, Class, and the Soul of the Nation. New Jersey: Princeton University Press, 1995.

Hurwitz, Jon and Mark Peffley. "Playing the Race Card in the Post-Willie Horton Era: The Impact of Racialized Code Words on Support for Punitive Crime Policy." Public Opinion Quarterly 69:1 (2005): 99-112.

Jansson, Bruce S. The Reluctant Welfare State: A History of American Social Welfare Policies. Pacific Grove, CA: Brooks/Cole Publishing Company, 2005.

Katz, Michael B. The Price of Citizenship: Redefining the American Welfare State. (2001) Philadelphia: University of Pennsylvania Press, 2008.

Katz Olson, Laura. The Politics of Medicaid. New York: Columbia University Press, 2010.

Kahlenberg, Richard D. “Fixing No Child Left Behind,” The Century Foundation. 2009. 11 Oct. 2010.

Lacy, Karyn R. Blue-Chip Black: Race, Class, and Status in the New Black Middle Class. Berkeley: University of California Press, 2007.

Langan, Patrick A. "Race of Prisoners Admitted to State and Federal Institutions, 1926-86." United States Department of Justice, Office Justice Programs, Bureau of Justice Statistics. 1991.

Lawrence, Jacob R. and Robert Y. Shapiro. Politicians Don't Pander: Political Manipulation and the Loss of Democratic Responsiveness. Chicago: University of Chicago Press, 2000.

Levendusky, Matthew. The Partisan Sort: How Liberals Became Democrats and Conservatives Became Republicans. Chicago: University of Chicago Press, 2009.

Lieberman, Robert C. Shaping Race Policy: The United States in Comparative Perspective. Princeton: Princeton University Press, 2007.

---. Shifting the Color Line: Race and the American Welfare State. (1998) Cambridge: Harvard University Press, 2001.

Lowi, Theodore J., Benjamin Ginsberg, and Kenneth A. Shepsle. American Government: Freedom and Power. (1990). New York: Norton and Company, 2006.

Malcolm X, By Any Means Necessary. 1970; New York: Pathfinder, 1992.

Marable, Manning and Kristin Clarke, eds. Barack Obama and African American Empowerment: The Rise of Black America's New Leadership. New York: Palgrave MacMillan, 2009.

Mendelberg, Tali. The Race Card: Campaign Strategy, Implicit Messages, and the Norm of Equality. Princeton: Princeton University Press, 2001.

National Federation of Independent Business et Al. v. Sebelius, Secretary of Health and Human Services 648 F. 3d 12352012.

Noble, Charles. Welfare As We Knew It: A Political History of the American Welfare State. New York: Oxford University Press, 1997.

Obama, Barack. "Prepared Remarks of President Barack Obama : Back to School Event." 08. Sept. 2009. Consulted 18. Nov. 2013. <http://www.whitehouse.gov/MediaResources/ PreparedSchoolRemarks/ >.

---. "Remarks by the President on Education Reform at the National Urban League Centennial Conference.” 29. Jul. 2010. Consulted 18. Nov. 2013. <http://www.whitehouse.gov/the-pressoffice/remarks-presdient-education-reform-national-urban-league-centennial-conference>. 
---. "Remarks by the President to the Hispanic Chamber of Commerce on a Complete and Competitive American Education," 10. March 2009. Consulted 18. Nov. 2013. <http:// www.whitehouse.gov/the-press-office/remars-president-united-states-hispanic-chambercommerces.

---. The Audacity of Hope. 2006. Edinburgh : Canongate Books, 2008.

Pearson, Robert W. "Social Statistics and an American Urban Underclass : Improving the Knowledge Base for Social Policy in the 1990s." Journal of the American Statistical Association 86, no. 414 (1991) : 504-12.

Persons, Georgia A. Dilemmas of Black Politics : Issues of Leadership and Strategy. New York: Harper Collins, 1993.

Reagan, Ronald. "Inaugural Address," January 20, 1981. Online by Gerhard Peters and John T. Woolley, The American Presidency Project. <http://www.presidency.ucsb.edu/ws/?pid=43130>.

---. State of the Union Address 1986. consulted 13.Jan.2014. <http://reagan2020.us/speeches/ state_of_the_union_1986.asp>.

Republican Party Platforms. "Republican Party Platform of 2000," July 31, 2000. Consulted 14. Jan. 2014. Online by Gerhard Peters and John T. Woolley, The American Presidency Project. <http:// www.presidency.ucsb.edu/ws/?pid=25849>.

---. "Republican Party Platform of 2004," August 30, 2004. Consulted 14. Jan. 2014. Online by Gerhard Peters and John T. Woolley, The American Presidency Project. <http:// www.presidency.ucsb.edu/ws/?pid=25850>.

Ricketts, Erol R., and Ronald B. Mincy. "Growth of the Underclass: 1970-1980." The Journal of Human Resources 25, no. 1 (1990): 137-45.

--- and Isable V. Sawhill. "Defining and Measuring the Underclass." Journal of Policy Analysis and Management 7, no. 2 (1988): 316-25.

Schmidtz, David and Robert E. Goodin. Social Welfare and Individual Responsibility: For and Against. Cambridge: Cambridge University Press, 1998.

Skocpol, Theda. Social Policy in the United States: Future Possibilities in Historical Perspective. New Jersey: Princeton University Press, 1995.

Sniderman, Paul M. and Edward G. Carmines. Reaching Beyond Race. Cambridge, Mass: Harvard University Press, 1997.

Somers, Margaret R. and Fred Block. "From Poverty to Perversity: Ideas, Markets, and Institutions over 200 Years of Welfare Debate," American Sociological Review 70:2 (2005): 260-287.

Starr, Paul. Remedy and Reaction: The Peculiar American Struggle over Health Care Reform. New Haven: Yale University Press, 2011.

Tesler, Michael and David O. Sears. Obama's Race: The 2008 election and the Dream of a Post-Racial America. Chicago: University of Chicago Press, 2010.

Thomas, Clarence. My Grandfather's Son: A Memoir, 2007; New York: Harper Perennial, 2008.

United States Supreme Court. National Federation of Independent Business et al. v. Sebelius, Secretary of Health and Human Services, et al. 28 June, 2012. Consulted 25. Jan. 2013. <http:// www.law.cornell.edu/supremecourt/text/11-393\#writing-11-393_SYLLABUS>.

Watts, Jerry and Nan Marie Astone. "The Personal Responsibility and Work Opportunity Act of 1996 by the $104^{\text {th }}$ Congress of the United States." Contemporary Sociology 26: 4 (1997): 409-415. 
Werner Cahalan, Margaret and Lee Anne Parsons. "Historical Corrections Statistics in the United States, 1850 -1984." United States Department of Justice, Bureau of Justice Statistics. Westat Inc., Rockville, Md. December 1986.

West, Cornel. Race Matters. 1993. New York: Vintage Books, 2001.

Wilson, William Julius. The Declining Significance of Race: Blacks and Changing American Institutions. $3^{\text {rd }}$ ed. 1978. Chicago: University of Chicago Press, 2012.

\section{NOTES}

1. Bruce Jansson, The Reluctant Welfare State: A History of American Social Welfare Policies (Pacific Grove, CA: Brooks/Cole Publishing Company, 2005) 304-5; 320.

2. Thomas B. Edsall and Mary D. Edsall, Chain Reaction: The Impact of Race, Rights, and Taxes on American Politics (New York: Norton and Company, 1992) 123; 131; 136.

3. Le pourcentage de prisonniers noirs dans les prisons d'Etat est passé de 35\% (toutes personnes de couleur confondues) en 1960 avec un âge médian de 27 à 43,5\% en 1979 (Noirs seuls) avec un âge médian de 24,9 ans. Werner Cahalan, Margaret and Lee Anne Parsons. "Historical Corrections Statistics in the United States, 1850 -1984." United States Department of Justice, Bureau of Justice Statistics. Westat Inc., Rockville, Md. December 1986. p.66; 68. Le pourcentage de Noirs dans les prisons d'Etat était de $43 \%$ en 1970 selon Langan, Patrick A. "Race of Prisoners Admitted to State and Federal Institutions, 1926-86." United States Department of Justice, Office Justice Programs, Bureau of Justice Statistics. 1991. p.5.

Le pourcentage de naissances hors mariage est passé de 34,9\% pour les Noirs (comparé à 4,8\% pour les Blancs) en 1965-9 à 43\% en 1970-4 (comparé à 6\% pour les Blancs) et à 51,7\% en 1975-9 (comparé à 8,2\% pour les Blancs). Akerlof, George A., Janet L. Yellen and Michael L. Katz. "An Analysis of Out-of-Wedlock Childbearing in the United States," The Quarterly Journal of Economics 111:2 (1996): 283.

4. William Julius Wilson, The Declining Significance of Race: Blacks and Changing American Institutions. $3^{\text {rd }}$ ed. (1978. Chicago: University of Chicago Press, 2012) 2; 22; 130. Wilson fait une démonstration très détaillée et fouillée de l'émergence de cettte underclass. Il faut cependant noter que le terme ne fait pas l'unanimité. Jennifer Hochschild, notamment, le conteste et préfère le terme de "the estranged poor".

Karyn R. Lacy, Blue-Chip Black: Race, Class, and Status in the New Black Middle Class. (Berkeley: University of California Press, 2007) 14.

La définition de la underclass se fait notamment par rapport à la localisation et concerne donc les populations pauvres au comportement qualifié de «dysfonctionnel» ou encore de «pathologique» qui vivent dans des zones urbaines de centre ville (ghetto) avec de fortes concentrations de ce type de comportements (criminalité, toxicomanie, grossesses chez les adolescentes, naissances hors-mariage, et forte proportion d'hommes adultes au chômage, voire hors du marché du travail). Ricketts, Erol R., and Ronald B. Mincy. "Growth of the Underclass: 1970-1980." The Journal of Human Resources 25, no. 1 (1990): 137.

Bien que la underclass ne représente qu'au plus $4 \%$ de la population totale (estimation approximative pour 1980), il y a eu une augmentation spectaculaire de familles vivant sous le seuil de la pauvreté entre 1959 et 1985, et ce notamment pour la population noire qui passe de $38 \%$ à $61 \%$. C'est surtout dans les grandes villes industrialisées du Nord-Est qu'on peut observer une concentration de la pauvreté : à New York City la population vivant dans des quartiers que l'on peut qualifier de ghettos a augmenté de 300\% entre 1970 et 1980. Pearson, Robert W. "Social Statistics and an American Urban Underclass: Improving the Knowledge Base for Social Policy in the 1990s." Journal of the American Statistical Association 86, no. 414 (1991): 504-5. 
C'est notamment cette augmentation spectaculaire et concentrée qui a contribué à la visibilité du phénomène.

5. Edsall p.11; 52; 101-2; 154; 211; 234-5.

Edward D. Berkowitz, America's Welfare State: From Roosevelt to Reagan (Baltimore: The Johns Hopkins University Press, 1991) 3-4.

6. Michael B. Katz, The Price of Citizenship: Redefining the American Welfare State (Philadelphia: University of Pennsylvania Press, 2008) 19.

Edsall 9.

Mark D. Brewer and Jeffrey M. Stonecash, Split: Class and Cultural Divides in American Politics (Washington: CQ Press, 2007) 10.

7. Martin Gilens, Why Americans Hate Welfare: Race, Media, and the Politics of Antipoverty Policy (Chicago: University of Chicago Press, 1999) 3.

8. Cornel West, Race Matters (1993. New York: Vintage Books, 2001) 157. Robert C. Lieberman, Shaping Race Policy: The United States in Comparative Perspective (Princeton: Princeton University Press, 2007) 4.

Pour une mise en perspective historique de ce phénomène, voir Feagin, Joe, R. White Party, White Government: Race, Class, and U.S. Politics. New York: Routledge, 2012.

9. Ralph Waldo Emerson, The Conduct of Life (1866. Boston: Ticknor and Fields, 1868) 71-110.

10. Jennifer L. Hochschild, Facing Up to the American Dream: Race, Class, and the Soul of the Nation (New Jersey: Princeton University Press, 1995) 31; 37; 39-40.

11. Georgia A. Persons, Dilemmas of Black Politics: Issues of Leadership and Strategy (New York: Harper Collins, 1993) 2.

12. Matthew Levendusky, The Partisan Sort: How Liberals Became Democrats and Conservatives Became Republicans (Chicago: University of Chicago Press, 2009).

13. John Gerring, Party Ideologies in America: 1828 - 1996 (Cambridge: Cambridge University Press, 1998) 239-41; 245.

14. Paul M. Sniderman and Edward G. Carmines Reaching Beyond Race (Cambridge, Mass: Harvard University Press, 1997) 74.

15. Laura Katz Olson, The Politics of Medicaid (New York: Columbia University Press, 2010) 12.

16. Eric M. Uslander and Thomas Zittel, "Comparative Legislative Behavior" p.392-408 in The Oxford Handbook of Political Science. Ed. Goodin, Robert E. (2009. New York: Oxford University Press, 2011) 393.

17. Edsall 69-70. Brewer and Stonecash 42.

18. Brewer and Stonecash 45. Charles Noble, Welfare As We Knew It: A Political History of the American Welfare State (New York: Oxford University Press, 1997) 111-2.

19. West 4.

20. John Harwood, "Don't Dare Call the Health Law 'Redistribution'," The New York Times, 23 Nov. 2013. Consulted 21 Apr. 2015 http://www.nytimes.com/2013/11/24/us/dont-dare-call-thehealth-law-redistribution.html. McCain a utilisé le terme "redistributor in chief".

21. Sniderman and Carmines 4. Persons 234.

22. Voir Edsall et Edsall, ou encore Tali Mendelberg.

23. Sniderman and Carmines 44-5; 78. En effet, les conservateurs s'opposent par principe à toute intervention étatique, sans avoir nécessairement des motivations racistes. La racialisation du discours vise donc primairement des libéraux ou des indépendants.

24. Brewer and Stonecash 10.

25. Brewer and Stonecash 117.

26. Persons 2.

27. Berkowitz p.xviii.

28. Robert C. Lieberman, Shifting the Color Line: Race and the American Welfare State (1998) Cambridge: Harvard University Press, 2001) x. 
29. Parmi les programmes visés se trouvaient principalement l'AFDC (Aid to Families with Dependent Children [allocations pour familles défavorisées]), le CETA (Comprehensive Employment and Training Act [Programme de formations pour adultes et recherche d'emploi]), et l'éligibilité pour Medicaid, notamment restreinte par le Omnibus Budget Reconciliation Act de 1981. Katz 57; 65-6; 264.

30. Edsall 15. Ces réticences viennent de deux facteurs: la peur de stigmatiser encore plus un groupe de personnes déjà fréquemment attaqué et l'idée d'avouer un échec des politiques sociales en admettant qu'il y a de graves problèmes parmi les populations les plus pauvres.

31. Margaret R. Somers and Fred Block, "From Poverty to Perversity: Ideas, Markets, and Institutions over 200 Years of Welfare Debate," American Sociological Review Vol. 70 No. 2 (2005): $265 ; 279$.

32. Edsall 121.

33. Brewer and Stonecash10; 15.

34. Wornie L. Reed, "Social Justice in the Age of Obama", p.193-214 in Race in the Age of Obama. Research in Race and Ethnic Relations. Vol.16. eds. Cunnigen, Donald and Marino A. Bruce (Bingley, UK: Emerald, 2010) 194.

35. Lawrence D. Bobo, "Somewhere between Jim Crow and Post-Racialism: Reflections on the Racial Divide in America Today.” Daedalus 140:2 (2011): 26.

36. Eduardo Bonilla-Silva, Racism without Racists: Colorblind Racism and the Persistence of Racial Inequality in America (Lanham, Md: Rowman and Littlefield, 2014) 259.

37. "Blacks Upbeat about Black Progress, Prospects: A Year After Obama's Election." Pew Research Center. January 21, 2010. http://www.pewsocialtrends.org/2010/01/12/blacks-upbeat-aboutblack-progress-prospects/ . Lors de ce sondage, $52 \%$ des Noirs ont répondu qu'ils pensaient que le manque de volonté et de responsabilité personnelle était une des causes de l'inégalité économique.

38. Jansson 273-5.

39. Jansson 304-5.

40. Françoise Coste, "Ronald Reagan and a New Partisan Identity: The Case of the Reagan Democrats." Communication, Colloque, "American Identities: In Relation and Interaction," Université Toulouse Le Mirail, 10-11 Juin 2010.

Il y avait $22 \%$ de Reagan-Democrats en 1980 . Reagan a obtenu les voix $54 \%$ de la classe ouvrière blanche et $47 \%$ des syndiqués. Le score de Jimmy Carter parmi ces électorats traditionnellement Démocrates a été inférieur.

41. Lieberman 3.

42. Martin Gilens, Why Americans Hate Welfare: Race, Media, and the Politics of Antipoverty Policy (Chicago: University of Chicago Press, 1999) 195.

43. Françoise Coste, "La longue marche de Ronald Reagan contre le mouvement des droits civiques : une croisade idéologique finalement vaine? » Communication, journée d'études « Mémoires du mouvement pour les droits civiques ». Université Toulouse-Le Mirail, 28 février 2014.

Françoise Coste, Reagan (Paris, France: Perrin, 2015).

44. Jansson 308; 332.

45. Katz 137-8.

46. Edsall 164; 178; 195. Les Républicains conservateurs ont fait de l'équilibre budgétaire un élément central de leur rhétorique, qui s'est traduit au niveau législatif par l'adoption du GrammRudman-Hollings Deficit Reduction Act en 1985 et du Balanced Budget Act de 1997.

47. Gerring 244. Les causes de discriminations citées dans le programme sont la race, la religion, le sexe, l'âge, l'origine nationale, l'héritage ethnique, l'orientation sexuelle, ou le handicap.

48. Ronald Reagan, State of the Union Address 1986. consulted 13.Jan.2014. <http://reagan2020.us/ speeches/state_of_the_union_1986.asp>. 
49. Theda Skocpol, Social Policy in the United States: Future Possibilities in Historical Perspective (New Jersey: Princeton University Press, 1995) 5.

La Social Security est un programme difficile à attaquer car il est perçu comme bénéficiant principalement aux personnes âgées blanches et le programme est vu comme quelque chose que l'on mérite en raison des contributions versées.

50. Martin Gilens a spécifiquement étudié le lien entre la représentation médiatique de la pauvreté par des personnes avec un visage noir depuis les années 1960 et l'association faite entre communauté noire et " undeserving poor».

51. Il y avait déjà depuis la présidence de Roosevelt une différence assez marquée entre les Démocrates du Nord et ceux du Sud, particulièrement concernant leurs attitudes vis-à-vis des minorités, en raison de la ségrégation légale imposée par les Démocrates dans les Etats du Sud après la Reconstruction. C'est après les élections présidentielles de 1960 et surtout 1964 que les Démocrates du Sud ont commencé à se tourner vers le Parti républicain.

52. Jansson 364. Edsall 12; 71; 138; 144; 211.

53. Ronald Reagan: «Inaugural Address, » January 20, 1981. Online by Gerhard Peters and John T. Woolley, The American Presidency Project. <http://www.presidency.ucsb.edu/ws/?pid=43130>.

54. Jon Hurwitz and Mark Peffley, "Playing the Race Card in the Post-Willie Horton Era: The Impact of Racialized Code Words on Support for Punitive Crime Policy." Public Opinion Quarterly 69:1 (2005): 101.

55. Jansson 325-8.

George H. W. Bush: «Inaugural Address, » January 20, 1989. Online by Gerhard Peters and John T. Woolley, The American Presidency Project. <http://www.presidency.ucsb.edu/ws/?pid=16610 >. La formulation utilisée par George H. W. Bush est : « The old solution, the old way, was to think that public money alone could end these problems. But we have learned that that is not so.»

56. Edsall 277.

57. Bill Clinton dans la préface à $\mathrm{Al}$ From, The New Democrats and the Return to Power (New York: Palgrave Macmillan, 2013) ix-x.

58. From 124.

59. Katz 20; 267. Noble 126-7. Edsall 52.

60. Edsall 176.

61. William J. Clinton: "Address Before a Joint Session of Congress on Administration Goals, » February 17, 1993. Online by Gerhard Peters and John T. Woolley, The American Presidency Project. Consulted 12. Dec. 2013. <http://www.presidency.ucsb.edu/ws/?pid=47232>.

62. Theodore J. Lowi, Benjamin Ginsberg, and Kenneth A. Shepsle. American Government: Freedom and Power. (1990. New York: Norton and Company, 2006) 212.

63. Katz Olson 2.

64. La façon de procéder de Bill Clinton avait aussi été critiquée par son propre parti. En effet, la réforme a été dirigée par la Maison Blanche avec la mise en place d'un comité de réforme. (Lawrence, Jacob R. and Robert Y. Shapiro. Politicians Don't Pander: Political Manipulation and the Loss of Democratic Responsiveness. Chicago: University of Chicago Press, 2000. p.91) Le fait que ce comité avait été placé sous la direction de la Première Dame Hillary Clinton avait encore plus exacerbé le sentiment du Congrès d'avoir été laissé de côté. Starr, Paul. Remedy and Reaction: The Peculiar American Struggle over Health Care Reform. New Haven: Yale University Press, 2011 p.81; 124). Obama a tiré les leçons de cet échec et remis l'élaboration de la réforme au Congrès.

65. Skocpol 8.

66. Jansson 381-3.

67. Jerry Watts and Nan Marie Astone, «The Personal Responsibility and Work Opportunity Reconciliation Act of 1996 by the $104^{\text {th }}$ Congress of the United States, »Contemporary Sociology Vol. 26 No.4 (1997): 409; 414. Smidtz et Goodin 104.

68. Jansson $405 ; 422$. 
69. Edsall 20; 152; 170.

70. Republican Party Platforms: « Republican Party Platform of 2000, » July 31, 2000. Consulted 14. Jan. 2014. Online by Gerhard Peters and John T. Woolley, The American Presidency Project $<$ http://www.presidency.ucsb.edu/ws/?pid=25849> 8.

Republican Party Platforms: « Republican Party Platform of 2004, » August 30, 2004. Consulted 14. Jan. 2014. Online by Gerhard Peters and John T. Woolley, The American Presidency Project. <http:// www.presidency.ucsb.edu/ws/?pid=25850 $>54$.

71. Ed.gov. The No Child Left Behind Act of 2001. U.S. Department of Education. 7 Jan. 2002. Web. 10 Oct. 2010. http://www2.ed.gov/nclb/overview/intro/execsumm.pdf>.

72. Richard D. Kahlenberg, "Fixing No Child Left Behind" The Century Foundation. 2009. 11 Oct. 2010. http://www.tcf.org/publications/education/agenda_rk.pdf.> 4. Il faut remarquer que le non-versement des fonds alloués révèle un décalage flagrant entre la rhétorique de la compassion et la politique réelle de G. W. Bush. Ceci est juste un point critiqué et critiquable parmi de nombreux autres. En revanche, la rhétorique de la réforme se voulait très constructive et non pas punitive.

73. Ed.gov. Recovery Act. U.S. Department of Education. N.d. Consulted. 16 Feb. 2011. http:// www.ed.gov/recovery>.

74. Republican party platform 2004 p.1; 62. L'hommage à Reagan dans le programme est très certainement lié en partie à son décès au mois de juin de la même année.

75. 2004 Bush platform 46-7.

76. George W. Bush, "State of the Union Address". 27 January 2001. Consulted 12.12.13 Online by Gerhard Peters and John T. Woolley, The American Presidency Project. http://www.let.rug.nl/usa/ presidents/george-w-bush/state-of-the-union-2001.php

77. History, Art, and Archives: United States House of Representatives. Barbara Jordan. Consulted 12. Dec. 2014. http://history.house.gov/People/Listing/J/JORDAN,-Barbara-Charline\%28J000266\%29/

From 76.

W.E.B. Du Bois "The Conservation of Races" in Back, Les and John Solomons, eds. Theories of Race and Racism: A Reader. $2^{\text {nd }}$ ed. (London: Routledge, 2009) 109.

W.E.B. Du Bois, The Soul of Black Folk (ed. Jim Manis, 2006. Electronic Classic Series. Hazelton, PA: PSU, 2014) 134.

78. Wilson 125.

79. Malcolm X, By Any Means Necessary (1970; New York: Pathfinder, 1992) 175.

Black Panther Party Platform 1966. Columbia American History Online. Consulted 12. Dec. 2014. http://caho-test.cc.columbia.edu/ps/10127.html

80. Clarence Thomas, My Grandfather's Son: A Memoir (2007; New York: Harper Perennial, 2008) 56-7; 73; 106-7; 130.

81. Barack Obama, The Audacity of Hope (2006; Edinburgh: Canongate Books, 2008) 52.

82. Liliana L. Herakova, Dijana Jelača, Razvan Sibii, and Leda Cooks, "Voicing Silence and Imagining Citizenship: Dialogues about Race and Whiteness in a "Postracial" Era," Communication Studies 62:4 (2011): 372-3.

83. Pour plus de détails concernant ces théories politiques noires voir, par exemple:

Cornel West, Race Matters (1993. New York: Vintage Books, 2001). Manning Marable and Kristin Clarke, eds. Barack Obama and African American Empowerment: The Rise of Black America's New Leadership (New York: Palgrave MacMillan, 2009). Georgia A. Persons, Dilemmas of Black Politics: Issues of Leadership and Strategy (New York: Harper Collins, 1993).

84. Barack Obama, « Prepared Remarks of President Barack Obama: Back to School Event, » 08. Sept. 2009. Consulted 18. Nov. 2013. < http://www.whitehouse.gov/MediaResources/ PreparedSchoolRemarks/ >. 
85. Barack Obama, «Remarks by the President on Education Reform at the National Urban League Centennial Conference," 29. Jul. 2010. Consulted 18. Nov. 2013. < http:// www.whitehouse.gov/the-press-office/remarks-presdient-education-reform-national-urbanleague-centennial-conference >.

86. Patient Protection and Affordable Care Act of 2010. Title 1 Subtitle F, Part I «Individual Responsibility " Sec. 1501 " Requirement to maintain minimum essential coverage, " and Title 1, Part A, Subpart II sec. 2713 « Coverage of preventive health services. »

87. Affordable Care Act. Title I subtitle F « Shared Responsibility » Part I

88. Affordable Care Act, Title I subtitle F « Shared Responsibility » Part II

89. Obama, Urban League Centennial Conference.

90. Obama, Audacity of Hope 63.

91. Barack Obama, «Remarks by the President to the Hispanic Chamber of Commerce on a Complete and Competitive American Education,» 10. March 2009. Consulted 18. Nov. 2013. $<$ http://www.whitehouse.gov/the-press-office/remars-president-united-states-hispanicchamber-commerce >.

Obama, Audacity of Hope 244.

92. Jansson 381-3.

93. Affordable Care Act. Title I Subtitle $C$ «Quality health insurance for all Americans » Part I «Health insurance Market Reforms,» and Subtitle D «Available coverage choices for all Americans » Part I «Establishment of qualified health plans » and Part IV « State flexibility to establish alternative programs » Sec. 1331. «State flexibility to establish basic health programs for low-income individuals not eligible for Medicaid. »

94. Affordable Care Act. Title I, Part I, Subpart A, Sec. 1401 and sec.1402. Cette clause prévoit des taux différents de subventions pour les foyers ayant des revenus entre $100 \%$ et $400 \%$ du niveau de pauvreté.

95. Smidtz and Goodin 107. Dans ces catégories on trouve par exemple les enfants, les personnes agées et les infirmes.

96. Edsall 278-9.

97. Gilens 2.

98. Obama, Back to School Event.

99. Obama, Urban League Centennial Conference.

100. National Federation of Independent Business et Al. v. Sebelius, Secretary of Health and Human Services 648 F. 3d 12352012.

\section{RÉSUMÉS}

Cet article étudie le transfert de la notion de «responsabilité » dans le discours politique américain de Reagan à Obama, en particulier dans la rhétorique entourant les questions sociales. Depuis les premières campagnes présidentielles de Reagan, l'attaque par les Républicains des politiques sociales lancées par les Démocrates s'est faite essentiellement sur le terrain de la notion de responsabilité. En effet, le discours républicain a alors créé l'image d'un vaste système d'aides sociales favorisant un comportement irresponsable chez ses bénéficiaires. Ce discours a culminé dans la défaite de la réforme de santé du président Bill Clinton en 1994. Au lieu d'une politique de santé plus généreuse, le Personal Responsibility and Work Opportunity Act de 1996 a été 
voté suite à la victoire électorale des Républicains au Congrès en novembre 1994. Cette nouvelle législation a durci les conditions d'accès à l'aide sociale et a lié les bénéfices de l'aide sociale à une compensation par le travail afin de responsabiliser les bénéficiaires, remplaçant ainsi l'idée de welfare par celle de workfare. Mais ce paysage rhétorique et idéologique a changé avec l'arrivée sur la scène politique de Barack Obama dans les années 2000. L'analyse de son discours montre comment il a habilement retravaillé la notion de responsabilité pour en faire plutôt un argument de défense de ses politiques sociales, comme par exemple obamacare ou la réforme de l'éducation. A l'aide de différents discours prononcés par Barack Obama, on comprend comment ce dernier lie la responsabilité des citoyens aux responsabilités du gouvernement afin de légitimer des politiques sociales plus généreuses.

This article analyzes the transfer of the notion of "responsibility" in American political discourse, particularly regarding social matters. Since the Reagan presidency, the Republicans have attacked social policies using the notion of personal responsibility, depicting the American system of social policies as favoring dependency, irresponsibility, and immorality. This discourse led to the failure of the Clinton health reform in 1994. Instead of a more generous health policy, the Personal Responsibility and Work Opportunity Act of 1996 was passed by the newly elected Republican Congress. The new legislation tied benefits to strong work requirements, thus substituting workfare to welfare. But this rhetorical and ideological framework has changed with Barack Obama. The analysis of his discourse shows that he uses the notion of responsibility to his own advantage, promoting more social policies such as the Affordable Care Act of 2010 or education reform. The examination of different speeches made by Barack Obama shows how he links the personal responsibility of the citizens to the government's responsibility in order to legitimize more generous and comprehensive social policies.

\section{INDEX}

Keywords : responsibility, social policies, Affordable Care Act, Obama, education, government Mots-clés : responsabilité, politique américaine, Barack Obama, politiques sociales, réforme de la santé

\section{AUTEURS}

\section{LEA STEPHAN}

ATER

Université de Toulouse-Jean Jaurès

lea.stephan@univ-tlse2.fr 